Check for updates

Cite this: J. Mater. Chem. A, 2021, 9 , 19625

Received 15th March 2021 Accepted 21st April 2021

DOI: 10.1039/d1ta02193a

rsc.li/materials-a

\section{Localized electron density modulation in conjugated polymer nanosheets for boosting photocatalytic $\mathrm{H}_{2}$ evolution $\dagger$}

A localized electron density modulation strategy is proposed to enhance the intrinsic surface catalytic proton reduction reaction by introducing dual pyridinic nitrogen $(\mathrm{N})$ atoms into the polymer skeleton. Theoretical calculations suggest that the increased localized electron density at the $\mathrm{N}$ site can enhance the electron density of states around the Fermi level, which would lower the energy barrier to $\mathrm{H}$ adsorption and activation during the photocatalytic hydrogen evolution (PHE) process. Moreover, the increased localized electron density also contributes to accelerating the photo-induced charge separation and transportation. Resultingly, an apparent quantum yield of $35.5 \%$ of the newly developed conjugated polymer (termed as COPPB-N2) can be achieved at a polymer concentration of $1 \mathrm{mg} \mathrm{mL}^{-1}$, which is the highest value achieved for conjugated microporous polymers to date. This work provides a new strategy through modulating the electronic properties of catalytically active sites for boosting the intrinsic photocatalytic performance.

\section{Introduction}

Photocatalytic hydrogen evolution (PHE) from water has been widely recognized as an ideal strategy to alleviate the energy crisis and environmental pollution. ${ }^{\mathbf{1 - 3}}$ Recently, conjugated polymers with remarkable advantages, such as versatile chemical composites, ${ }^{4-6}$ tunable molecular structures, ${ }^{7,8}$ and high thermal/chemical stability, ${ }^{\mathbf{9 , 1 0}}$ have attracted extensive interest for synthesizing efficient hydrogen evolution photocatalysts. The pioneer conjugated polymer photocatalyst poly( $p$-phenylene) exhibited a low $\mathrm{H}_{2}$ evolution rate $(\lambda \geq 290 \mathrm{~nm})$ in $1985,{ }^{11}$ but it inspired scientists to look further into developing novel polymer-based photocatalysts. Enormous efforts have been devoted to improving PHE performance mainly via three crucial approaches, i.e., by enhancing (i) the visible light absorption,

State Key Laboratory of Organic-Inorganic Composites, Beijing University of Chemical Technology, Beijing 100029, P. R. China. E-mail: xiangzh@mail.buct.edu.cn

$\dagger$ Electronic supplementary information (ESI) available. See DOI: 10.1039/d1ta02193a (ii) charge carrier separation, and (iii) surface catalytic reaction. ${ }^{12}$ Most recently, numerous reported studies mainly focused on the first two approaches. The visible light absorption can be increased via the copolymerization method with large planar conjugated monomers and different substituentmodified cross-linkers. ${ }^{13-16}$ Meanwhile, the photo-induced charge recombination can be suppressed by fabricating donor-acceptor structures, ${ }^{17-20}$ building organic heterojunctions, ${ }^{21}$ or reducing conjugated polymers into polymer dots (Pdots). ${ }^{22-24}$ However, studies on boosting the intrinsic surface catalytic activity have rarely been performed, and the poor surface catalytic activity is a critical obstacle to further improving the PHE efficiency of conjugated polymers. Loading cocatalysts on the surface of polymer photocatalysts is considered to be the most common way to reduce the energy barrier for boosting the sluggish surface proton reduction reaction, ${ }^{25-30}$ but how to improve their intrinsic surface catalytic activity is still a great challenge.

Alternatively, introducing heteroatoms into the skeleton of conjugated polymers can adjust the electronic configuration of the relevant reactive sites to improve catalytic performance..$^{31-35}$ For instance, doping $\mathrm{N}$ into a metal sulfide is carried out to modulate the electron density of the S site, via the interaction between the $2 \mathrm{p}$ orbital of the $\mathrm{N}$ atom and $2 \mathrm{~d}$ orbital of transition metals, to facilitate its surface catalytic proton reduction for electrocatalytic hydrogen evolution. ${ }^{36}$ After introducing pyridinic $\mathrm{N}$ into poly(phenylene) derivatives, the apparent quantum yield (AQY) can be dramatically enhanced from $0.4 \%$ to $6.7 \%$ at $420 \mathrm{~nm} \cdot{ }^{33}$ However, the surface catalytic activities are still not satisfactory and need to be further improved.

Herein, we newly developed a localized electron density modulation strategy to enhance the intrinsic surface catalytic proton reduction reaction by introducing dual pyridinic $\mathrm{N}$ atoms into a conjugated polymer nanosheet (termed as COP-PBN2). As a control, we also synthesized a fully conjugated polymer nanosheet without $\mathrm{N}$ doping (termed as COP-PB) and theoretically developed another polymer doped with only one pyridinic $\mathrm{N}$ in the skeleton (denoted as COP-PB-N1). The $\mathrm{sp}^{2}$ 
occupied orbital of the pyridinic $\mathrm{N}$ with lone-pair electrons can interact with the empty 1 s orbital of $\mathrm{H}$ which makes pyridinic $\mathrm{N}$ serve as a reactive site for proton reduction. More importantly, the introduced dual $\mathrm{N}$ atoms in COP-PB-N2 can increase the electron density around the $\mathrm{N}$ site, which will further strengthen the electron density of states at the Fermi level and thus lower the energy barrier to the formation of intermediate products during the PHE process. Consequently, the newly developed COP-PB-N2 shows a visible-light-driven hydrogen evolution rate of $51.5 \mu \mathrm{mol} \mathrm{h}{ }^{-1}$ which is 5-fold higher than that of undoped COP-PB, and achieves an apparent quantum yield (AQY) of $35.5 \%$ at $400 \mathrm{~nm}$ exceeding that of the state-of-the-art conjugated microporous polymer photocatalysts and even being comparable to that of the benchmark inorganic photocatalysts.

\section{Results and discussion}

The theoretical models of the three fully conjugated polymers in this work, i.e., COP-PB, COP-PB-N1 and COP-PB-N2, are shown in Fig. S1. $\uparrow$ The partial charge density image (Fig. 1a) illustrates that the increased localized electron density is mainly concentrated around pyridinic N. Moreover, the Bader effective charge (Fig. S2 and S3†) clearly reveals that the introduction of dual pyridinic $\mathrm{N}$ can induce the redistribution of surface charge and the $\mathrm{N}$ atom of COP-PB-N2 at site 1 is more negative than that of COP-PB-N1 at the same position, which would enable the pyridinic $\mathrm{N}$ to more easily anchor hydrogen atoms to form intermediate configurations. In order to clearly show the influence of localized electron density change on hydrogen adsorption which is a crucial step to determine the catalytic reaction rate, ${ }^{37,38}$ we calculate the charge density difference of the optimized adsorption configurations based on density functional theory (DFT), presented in Fig. 1b and S4. $\dagger$ The interaction between adsorbed $\mathrm{H}$ and polymers can be strengthened when introducing pyridinic $\mathrm{N}$ into conjugated polymers. The COP-PB-N2 has the maximum number of electrons transferred from polymers to adsorbed $\mathrm{H}$, which is beneficial to the $\mathrm{H}$ activation. ${ }^{39}$

As shown in Fig. S5, $\uparrow$ the electron density of states of COPPB-N2 at the Fermi level is the largest among the three samples due to the increased localized electron density on pyridinic $\mathrm{N}$ atoms, which contributes to the lower energy barrier to intermediate product formation during the PHE process. ${ }^{\mathbf{4 0 , 4 1}}$ Beyond that, the electron localization function (ELF) also confirms that the interaction of chemisorbed N-H on COP-PB$\mathrm{N} 2$ is stronger than that on COP-PB-N1, which might lead to a more efficient proton reduction (Fig. 1c). Furthermore, due to the absence of one atom in the steric structure, the steric hindrance to chemisorbed $\mathrm{N}-\mathrm{H}$ in COP-PB-N2 is a little smaller than that in COP-PB-N1 (Fig. 1c), which is favourable for $\mathrm{H}$ adsorption.

As mentioned above and emphasized in the reported $\mathrm{N}$ doped polymers, ${ }^{5,17,33}$ our theoretical screening finds that the
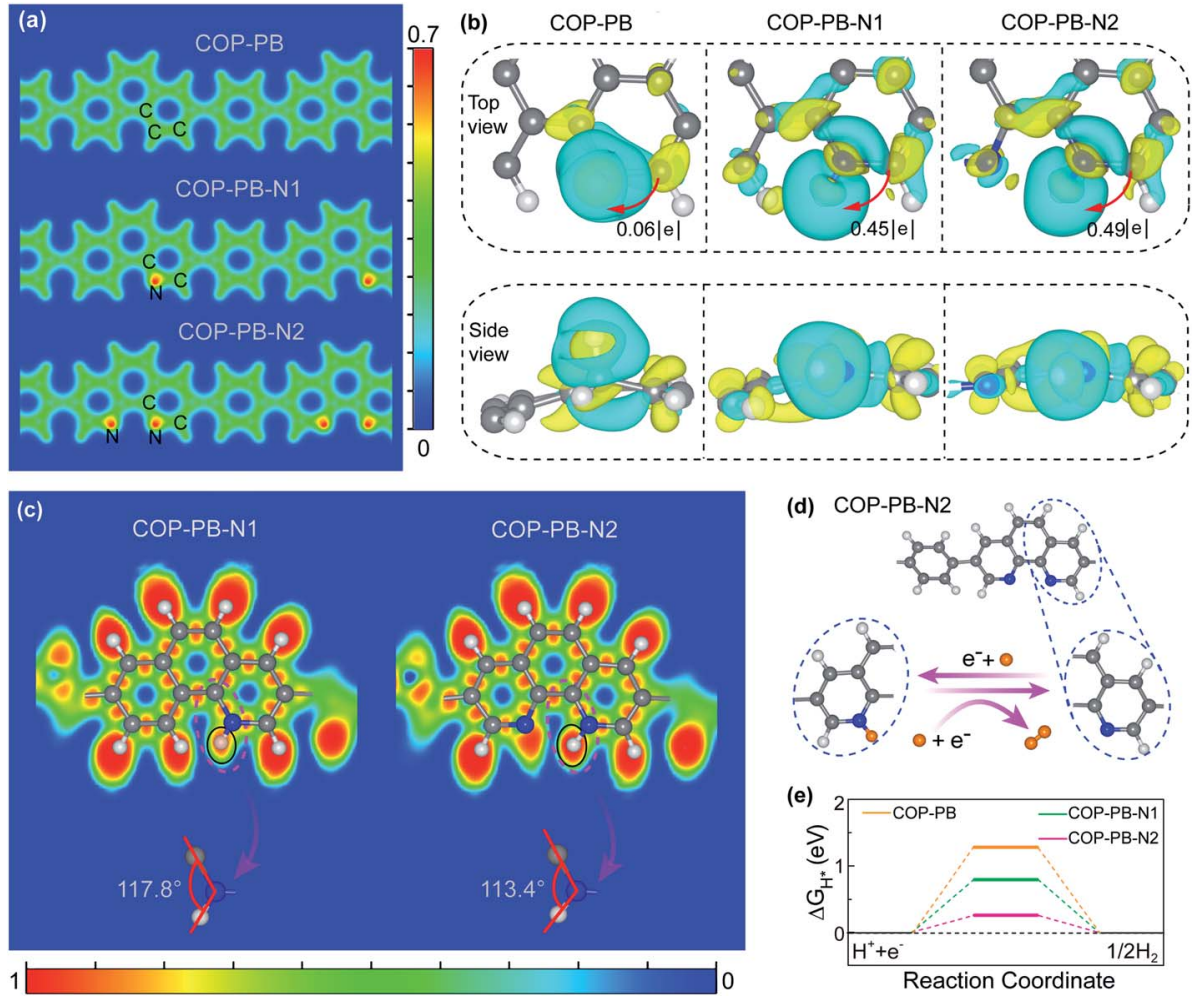

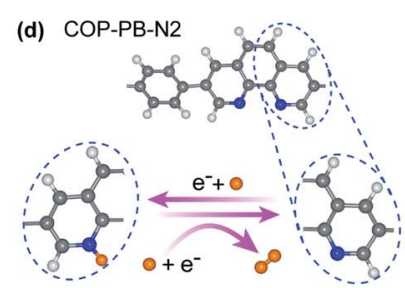

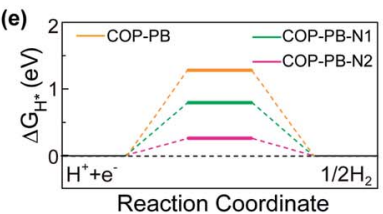

Fig. 1 (a) Partial charge density of the polymers. (b) The charge density differences of $\mathrm{H}$ chemisorbed on different polymers; cyan and yellow represent charge depletion and accumulation, respectively. The iso-surface value is 0.002 e $\AA^{-3}$ and the Fermi level is set to be 0 eV. (c) The electron localization function (ELF); the bond angles of carbon-nitrogen- $\mathrm{H}$. (d) The $\mathrm{H}_{2}$ evolution process (C grey, N blue, H from COP-PB-N2: white, $\mathrm{H}$ from water: orange). (e) The calculated hydrogen evolution reaction energy profile. 
pyridinic $\mathrm{N}$ in the COP-PB-N2 skeleton is confirmed as the surface catalytic site for PHE (vide infra). The catalytic process of $\mathrm{H}$-adsorption-reduction to $\mathrm{H}_{2}$ is shown in Fig. 1d, i.e., the hydrogen atom from water is first bonded with pyridinic $\mathrm{N}$ from COP-PB-N2 to become activated $\mathrm{H}$, which will react with another $\mathrm{H}$ from water to produce hydrogen. Furthermore, the calculated Gibbs free energy $\left(\Delta G_{\mathrm{H}^{*}}\right)$ of COP-PB, COP-PB-N1 and COP-PB$\mathrm{N} 2$, which is a good descriptor for evaluating the catalytic activity, $^{42}$ exhibits different values ranging from $0.26 \mathrm{eV}$ to $1.28 \mathrm{eV}$ (Fig. 1e). The $\Delta G_{\mathrm{H}^{*}}$ of COP-PB is relatively far from the optimal value of $\Delta G_{\mathrm{H}^{*}}=0 \mathrm{eV}$, indicating an inherently low catalytic activity for the hydrogen evolution reaction. ${ }^{\mathbf{4 3 , 4 4}}$ Meanwhile, the $\Delta G_{\mathrm{H}^{*}}$ of the $\mathrm{N}$ site from COP-PB-N2 is only $0.26 \mathrm{eV}$ which is much smaller than the $\mathrm{N}$ site $(0.80 \mathrm{eV})$ at the same position in COP-PB-N1 (Fig. 1e). Hence, COP-PB-N2 demonstrates the highest intrinsic catalytic activity for $\mathrm{PHE}$ compared with COP-PB and COP-PB-N1.

The as-prepared polymers of COP-PB and COP-PB-N2 were synthesized via a Suzuki cross-coupling reaction with corresponding monomers 1,4-phenylenebisboronic acid, 2,7-dibromophenanthrene and 3,8-dibromophenanthroline (Fig. 2a). The characterization results of FT-IR spectra and ${ }^{13} \mathrm{C}$ solid-state NMR spectra confirm the successful synthesis of COP-PB and COP-PB-N2 (Fig. S10 and S11 $\dagger$ ). ${ }^{45}$ Additionally, the powder X-ray diffraction (PXRD) (Fig. S12 $\dagger$ ) reveals the typical quasicrystalline properties of both polymers. ${ }^{33}$ The transmission electron microscope images (Fig. $2 b$ and c) of the two assynthesized polymers exhibit a nanosheet texture along with atomic force microscopy. The thickness of COP-PB-N2 and COP$\mathrm{PB}$ nanosheets is determined to be 8 and $4 \mathrm{~nm}$, respectively (Fig. 2d and e). The thin sheet-like structure can facilitate charge transfer to the conjugated polymer surface rapidly. ${ }^{\mathbf{4 6 , 4 7}}$

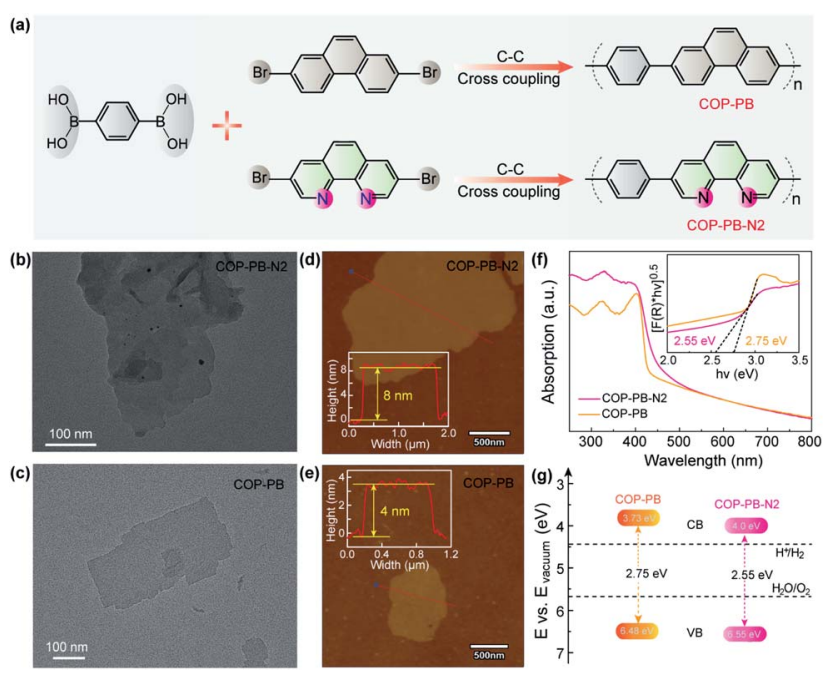

Fig. 2 (a) Schematic illustration for the synthesis of COP-PB and COPPB-N2. ( $b$ and $c$ ) Transmission electron microscopy (TEM) images of COP-PB-N2 and COP-PB. ( $d$ and e) Atomic force microscope (AFM) images of COP-PB-N2 and COP-PB (the insets show the height profiles). (f) UV-vis diffuse reflectance spectra (the inset shows the Tauc plots of COP-PB-N2 and COP-PB). (g) The diagram of electronic band structures for COP-PB and COP-PB-N2.
As shown in Fig. 2f, a broad visible light absorption of COP$\mathrm{PB}$ ranging from 400 to $600 \mathrm{~nm}$ was measured by UV-vis reflectance spectra in the solid state. The absorption of COPPB-N2 is enhanced owing to the extended $\pi$-conjugation caused by the increased electron delocalization. Correspondingly, the bandgaps are determined to be 2.75 and $2.55 \mathrm{eV}$ for COP-PB and COP-PB-N2 with the Kubelka-Munk functions from the absorption spectra (Fig. 2g). Meanwhile, ultraviolet photoelectron spectroscopy (UPS) determined the valence band (VB) to be $1.70 \mathrm{eV}$ and $1.85 \mathrm{eV}$ for COP-PB and COP-PB-N2, respectively, below the Fermi level $\left(E_{\mathrm{F}}\right)$ (Fig. S13 $\dagger$ ). The work functions $(\Phi)$ of COP-PB and COP-PB-N2 are calculated to be 4.78 and $4.70 \mathrm{eV}$ based on the secondary electron cut-off (Fig. S14 $\dagger$ ). Therefore, the VB of COP-PB and COP-PB-N2 turn out to be 6.48 and $6.55 \mathrm{eV}$ correlated with the vacuum level. Consequently, the bandgaps along with the corresponding conduction band (CB) positions could endow the conjugated polymers with suitable thermodynamic driving force for the proton reduction reaction (Fig. $2 \mathrm{~g}$ ).

The PHE performance of the as-synthesized polymers is studied in a mixture of water, methanol and triethylamine (TEA), where the methanol accelerates the mixing of water and TEA. ${ }^{48}$ The COP-PB shows a moderate PHE of $11 \mu \mathrm{mol} \mathrm{h}{ }^{-1}$ under visible light ( $\lambda \geq 400 \mathrm{~nm}$ ). Encouragingly, after introducing dual pyridinic $\mathrm{N}$ into the skeleton of COP-PB, the PHE performance of COP-PB-N2 can be elevated to $51.5 \mu \mathrm{mol} \mathrm{h} \mathrm{h}^{-1}$ (Fig. 3a). Significantly, the apparent quantum yield (AQY) of COP-PB-N2 reaches $22.1 \%$ at $400 \mathrm{~nm}$ and can be further elevated to $35.5 \%$ when the polymer concentration is increased from $0.25 \mathrm{mg} \mathrm{mL}^{-1}$ to $1 \mathrm{mg} \mathrm{mL}{ }^{-1}$, which remarkably exceeds the state-of-the-art conjugated microporous polymers (Fig. $3 \mathrm{~b}$ and g) and is even comparable to the benchmark inorganic photocatalysts. ${ }^{49,50}$ Although the photocatalytic $\mathrm{H}_{2}$ evolution rate is high in the field of conjugated microporous polymers, it is not enough to meet the practical applications, which needs to be further improved.

The active site screening in COP-PB and COP-PB-N2 confirms the pyridinic $\mathrm{N}$ to be the reactive site (Fig. $3 \mathrm{c}$ and $\mathrm{S} 15 \dagger$ ). The optimal $\Delta G_{\mathrm{H}^{*}}$ of the carbon site is much larger than that of the $\mathrm{N}$ site, demonstrating the pyridinic $\mathrm{N}$ to be the reactive site, which is ascribed to the suitable electron configuration of pyridinic N. As illustrated in Fig. 3d and e, the electron structure of the pyridinic $\mathrm{N}$ atom is $2 \mathrm{~s}^{2} 2 \mathrm{p}^{3}$ and the $\mathrm{sp}^{2}$ hybridization of these orbitals will generate three $\mathrm{sp}^{2}$ hybrid orbitals, two of which are half filled and the third one is occupied with a pair of electrons. Both the $\mathrm{p}_{z}$ orbital of the pyridinic $\mathrm{N}$ atom and the other five $\mathrm{p}_{z}$ orbitals of $\mathrm{C}$ atoms will result in a large closed $\pi$ bond. The $\mathrm{sp}^{2}$ hybrid orbital with a pair of electrons will be conducive to bonding with the $\mathrm{H} 1$ s orbital and will thus boost the $\mathrm{H}$ activation. Therefore, the photocatalytic hydrogen production process of COP-PB-N2 can be illustrated as in Fig. $3 \mathrm{f}$. The photo-excited electrons and holes inside conjugated polymers can rapidly transfer to the surface and subsequently react with adsorbed $\mathrm{H}$ and TEA, respectively.

The control experiments are carried out (Fig. S16†) in order to avoid the influence of the above mixed solvent. The pristine $\mathrm{H}_{2} \mathrm{O} /$ methanol/TEA solvent cannot generate hydrogen with or 

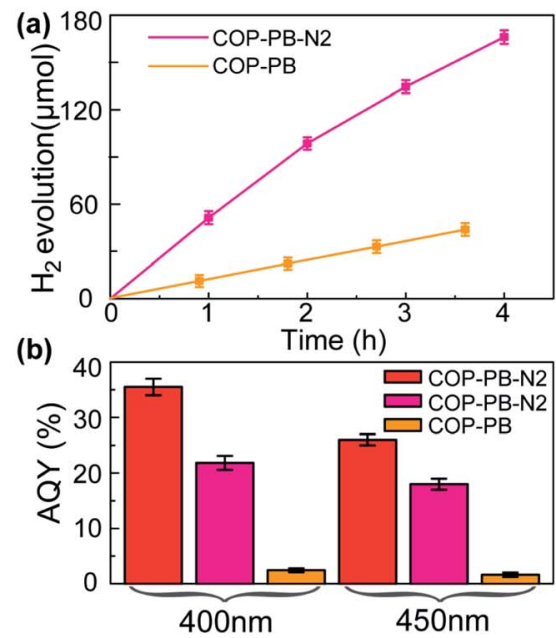

(c)

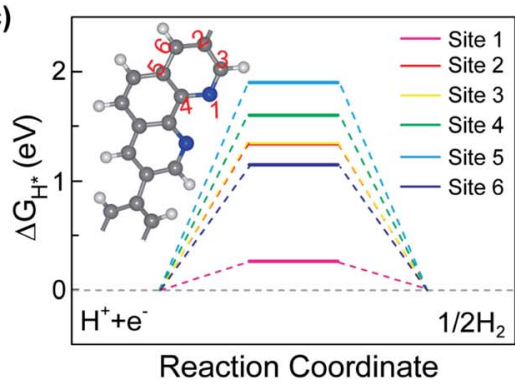

(g) (d)

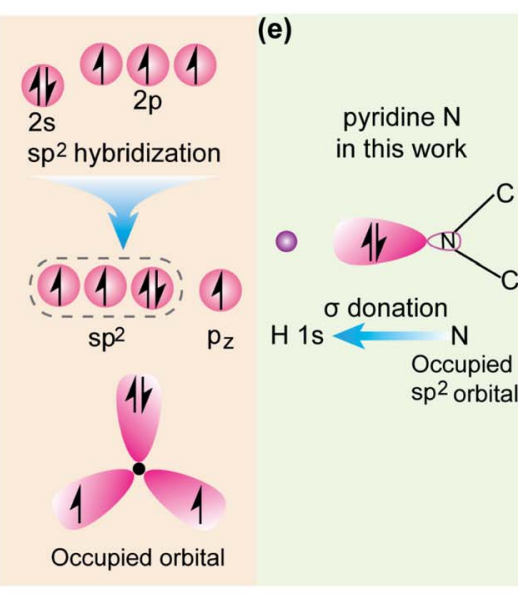

(f)

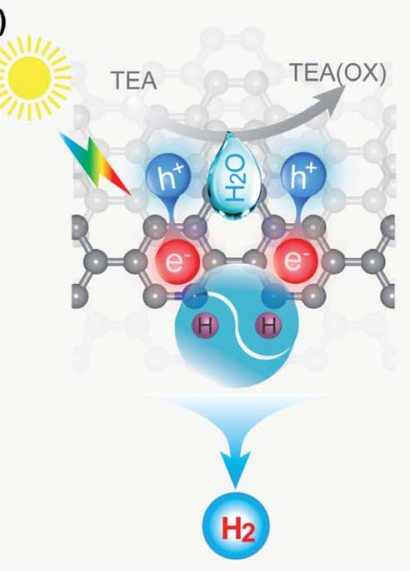

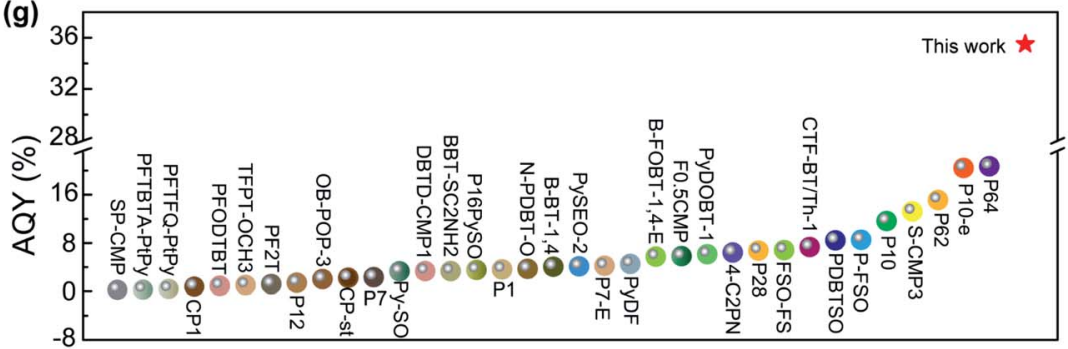

Fig. 3 (a) Visible-light-driven hydrogen production of COP-PB-N2 and COP-PB ( $\lambda \geq 400 \mathrm{~nm}$ ). (b) Apparent quantum yield (AQY) of COP-PB-N2 and COP-PB; red represents a polymer concentration of $1 \mathrm{mg} \mathrm{mL}^{-1}$, pink and orange represent a polymer concentration of $0.25 \mathrm{mg} \mathrm{mL}^{-1}$. (c) The calculated hydrogen evolution reaction energy profile of different sites on COP-PB-N2. The inset is the structure of COP-PB-N2. (d) The electronic configuration of a pyridinic $\mathrm{N}$ atom and a $\mathrm{N}$ atom with $\mathrm{sp}^{2}$ hybridization. (e) Illustration of $\mathrm{H}$ bonding to a pyridinic $\mathrm{N}$ atom on $\mathrm{COP}$ - $\mathrm{PB}-$ N2. (f) The illustration of photocatalytic hydrogen production with COP-PB-N2 under visible light irradiation. (g) The AQY performance comparison between COP-PB-N2 and other reported conjugated polymer photocatalysts; the star represents COP-PB-N2 with a polymer concentration of $1 \mathrm{mg} \mathrm{mL}^{-1}$ at $400 \mathrm{~nm}$ (most results are at $400 \mathrm{~nm}$ or $420 \mathrm{~nm}$ and the detailed irradiation conditions can be found in Table $\mathrm{S} 2 \uparrow$ ).

without visible light irradiation and no hydrogen is measured for the two conjugated polymers in the $\mathrm{H}_{2} \mathrm{O}$ /methanol/TEA solvent in the dark. Meanwhile, hydrogen cannot be produced with the mixture of water/methanol or pure water, but can be evolved when using a mixture of TEA/water. These results demonstrate that methanol acts as a co-solvent and TEA is a hole scavenger rather than the polymer itself. The FT-IR spectrum indicates that the skeleton of COP-PB-N2 does not fall apart after photocatalysis (Fig. S17†). In addition, we have also synthesized three different batches of samples under the same experimental parameters to ensure the repeatability of the two polymer photocatalysts. Each of the polymers exhibits an almost identical $\mathrm{H}_{2}$ production rate under the same photocatalytic conditions, manifesting the outstanding reproducibility of the as-prepared polymers (Fig. S18†). It is imperative to point out that the COP-PB has more residual palladium than COP-PB-N2 (Table S3†); however, it has much lower photocatalytic performance, which suggests that the residual Pd does not play a crucial role in improving photocatalytic $\mathrm{H}_{2}$ evolution. Meanwhile, we have also carried out photocatalytic cyclic testing of COP-PB-N2, which shows that the photocatalytic $\mathrm{H}_{2}$ evolution rate has been significantly decreased after one cyclic testing (Fig. S19†). To find the reason why the photocatalytic performance is dramatically decreased, we further conducted Xray photoelectron spectroscopy (XPS) measurements (Fig. S20†). The slight shift of nitrogen peaks indicates that the state of some pyridinic nitrogen atoms may have been changed after photocatalytic $\mathrm{H}_{2}$ evolution. This result confirms that the pyridinic nitrogen atoms are the main catalytic sites. Additionally, the COP-PB-N2 nanosheets tend to aggregate after the photocatalytic cyclic test (Fig. S21 $\dagger$ ), which might be another reason for the photocatalytic performance decline. Furthermore, previous research has also confirmed that the residual palladium can play a partial catalytic role, but is not the major factor for maximizing the photocatalytic hydrogen evolution rate.,51-54

Additionally, the photo-excited charge separation and transportation are also fully investigated. The behaviour of photo-induced electrons and holes is detected via electron paramagnetic resonance (EPR) assisted by 2,2,6,6-tetramethyl-Lpiperidine- $N$-oxyl (TEMPO). ${ }^{55}$ The obvious triplet peak from Fig. 4a in the dark is the characteristic signal of TEMPO, and then the TEMPO will be reacted with photo-induced electrons $\left(\mathrm{e}^{-}\right)$or holes $\left(\mathrm{h}^{+}\right)$to produce TEMPOH or TEMPO ${ }^{+}$in different solutions (Fig. S22 $\uparrow$ ). Subsequently, the decreased intensity of the triplet peaks at intervals of time confirms that the polymers can really produce electrons $\left(\mathrm{e}^{-}\right)$and holes $\left(\mathrm{h}^{+}\right)$under visible 

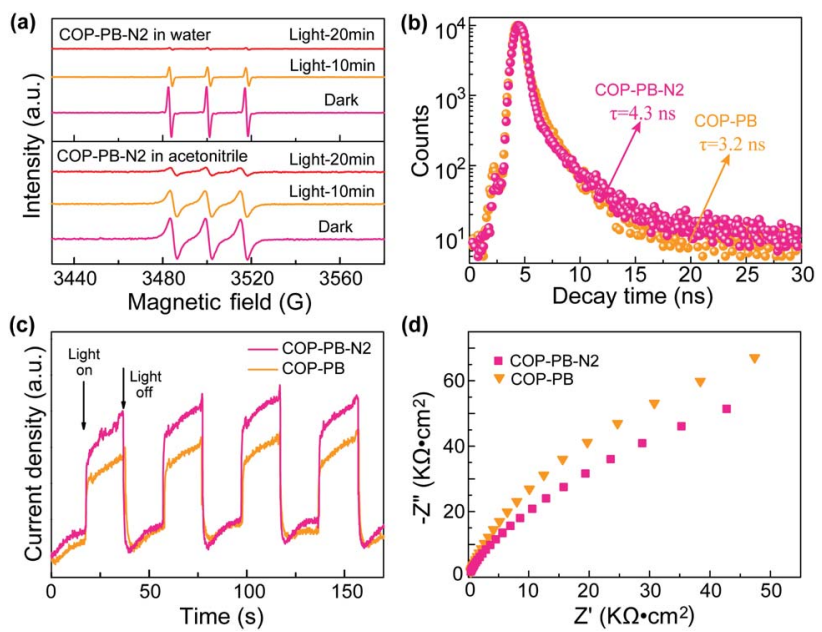

Fig. 4 (a) EPR curves for monitoring photo-excited electrons and holes. (b) Time-resolved transient PL decay spectra. (c) On-off photocurrent response under visible light or in the dark. (d) Electrochemical impedance spectroscopy Nyquist plots.

light irradiation. ${ }^{56}$ Moreover, the time-resolved PL decay plots (Fig. 4b) of the above polymers have been fitted with a multiexponential kinetic function and the average lifetime has been calculated in the ESI (Table S1 $\dagger$ ). ${ }^{57,58}$ A slightly longer lifetime of COP-PB-N2 than COP-PB might reveal the improved separation of photo-excited electron-hole pairs. ${ }^{43,59,60}$ The transient photocurrent intensity of COP-PB-N2 under on-off visible light irradiation is slightly stronger than that of COP-PB, which confirms the presence of a few more photo-excited charge carriers due to the relatively enhanced visible light absorption. ${ }^{61,62}$ Additionally, we conducted a contact angle experiment to explore the wettability as shown in Fig. S23. $\dagger$ After introducing pyridinic $\mathrm{N}$ into the polymers, the surface affinity of COP-PB-N2 with $\mathrm{H}_{2} \mathrm{O}$ or $\mathrm{H}_{2} \mathrm{O} / \mathrm{MeOH} / \mathrm{TEA}$ is enhanced contributing to boosting the interfacial charge transfer. ${ }^{63}$ Furthermore, on the basis of electrochemical impedance spectroscopy (Fig. 4d), the smaller Nyquist semicircle curve of COP-PB-N2 than COP-PB also demonstrates the slightly improved interfacial charge transfer. ${ }^{6,65}$ These results reveal that the separation of photo-induced electron-hole pairs can also be enhanced after introducing dual pyridinic $\mathrm{N}$ apart from improving interfacial charge transfer, and both of them synergistically improve PHE performance.

\section{Conclusions}

In summary, we have successfully developed a highly efficient conjugated polymer towards PHE via a localized electron density modulation strategy using dual pyridinic $\mathrm{N}$ atoms. The newly developed COP-PB-N2 has achieved an excellent photocatalytic $\mathrm{H}_{2}$ production rate of $51.5 \mu \mathrm{mol} \mathrm{h}{ }^{-1}$. An AQY of $35.5 \%$ can be achieved at a polymer concentration of $1 \mathrm{mg} \mathrm{mL} \mathrm{mL}^{-1}$, which exceeds that of the state-of-the-art conjugated microporous polymer photocatalysts. Our theoretical calculations and experiments suggest that the $\mathrm{sp}^{2}$ hybridized orbital of pyridinic $\mathrm{N}$ with lone-pair electrons is favourable for facilitating
$\mathrm{H}$ adsorption and activation. The improved localized electron density of COP-PB-N2 plays a decisive role in increasing the electron density of states at the Fermi level, which significantly lowers the energy barrier to intermediate product formation during the PHE process. Meanwhile, the optimal Gibbs free energy $\left(\Delta G_{\mathrm{H}^{*}}\right)$ of COP-PB-N2 demonstrates its superior intrinsic surface catalytic activity. Moreover, the increased localized electron density is also conducive to enhancing the charge separation and transfer. Therefore, the strategy of localized electron density modulation offers new avenues to develop more highly efficient conjugated polymers for photocatalytic or even other catalytic reactions.

\section{Conflicts of interest}

There are no conflicts to declare.

\section{Acknowledgements}

This work was supported by the Beijing Natural Science Foundation (JQ19007), NSF of China (21676020 and 21922802), the Young Elite Scientists Sponsorship Program by CAST (2017QNRC001), the Talent Cultivation and Open Project (OIC201801007) of State Key Laboratory of Organic-Inorganic Composites, "Double-First-Class" Construction Projects (XK180301 and XK1804-02), and the Distinguished Scientist Program at BUCT (buctylkxj02).

\section{Notes and references}

1 Y. Wang, A. Vogel, M. Sachs, R. S. Sprick, L. Wilbraham, S. J. A. Moniz, R. Godin, M. A. Zwijnenburg, J. R. Durrant, A. I. Cooper and J. W. Tang, Nat. Energy, 2019, 4, 746-760.

2 S. Chen, T. Takata and K. Domen, Nat. Rev. Mater., 2017, 2, 17050-17067.

3 Z. Wang, C. Li and K. Domen, Chem. Soc. Rev., 2019, 48, 2109-2125.

4 W. Zhong, J. Liang, S. Hu, X. F. Jiang, L. Ying, F. Huang, W. Yang and Y. Cao, Macromolecules, 2016, 49, 5806-5816.

5 P. B. Pati, G. Damas, L. Tian, D. L. A. Fernandes, L. Zhang, I. B. Pehlivan, T. Edvinsson, C. M. Araujo and H. Tian, Energy Environ. Sci., 2017, 10, 1372-1376.

6 L. Li, R. G. Hadt, S. Yao, Q. Wu, B. Pandit, L. X. Chen and L. Yu, Chem. Mater., 2016, 28, 5394-5399.

7 X. H. Zhang, X. P. Wang, J. Xiao, X. Ding, Y.-G. Xiang and H. Chen, J. Catal., 2017, 350, 64-71.

8 C. Cheng, X. Wang, Y. Lin, L. He, J. X. Jiang, Y. Xu and F. Wang, Polym. Chem., 2018, 9, 4468-4475.

9 C. Yang, B. C. Ma, K. Landfester, K. A. Zhang and X. Wang, Angew. Chem., Int. Ed., 2016, 55, 9202-9206.

10 L. Li, Z. Cai, Q. Wu, W. Y. Lo, N. Zhang, L. X. Chen and L. Yu, J. Am. Chem. Soc., 2016, 138, 7681-7686.

11 A. K. Shozo Yanagida, K. Mizumoto and K. Yoshino, J. Chem. Soc., Chem. Commun., 1985, 474-475.

12 J. Ran, J. Zhang, J. Yu, M. Jaroniec and S. Z. Qiao, Chem. Soc. Rev., 2014, 43, 7787-7812. 
13 C. Dai, S. Xu, W. Liu, X. Gong, M. Panahandeh-Fard, Z. Liu, D. Zhang, C. Xue, K. P. Loh and B. Liu, Small, 2018, 14, 1801839.

14 Y. Liu and Z. Xiang, ACS Appl. Mater. Interfaces, 2019, 11, 41313-41320.

15 L. Wang, Y. Wan, Y. Ding, S. Wu, Y. Xiong, X. Wu, J. Yang and H. Xu, Adv. Mater., 2017, 29, 2428-2435.

16 Z. Wang, X. Yang, T. Yang, Y. Chen, J. H. Zeng, C. Yan, F. Huang and J. X. Jiang, ACS Catal., 2018, 8, 8590-8596.

17 Y. Xiang, X. Wang, L. Rao, P. Wang, X. Zhang, S. Wang, H. Chen and Y. Zhu, ACS Energy Lett., 2018, 3, 2544-2549.

18 Y. Liu, Z. Liao, X. Ma and Z. Xiang, ACS Appl. Mater. Interfaces, 2018, 10, 30698-30705.

19 Y. Xu, C. Zhang, F. Wang and J. X. Jiang, Appl. Catal., B, 2018, 228, 1-9.

20 J. Yu, X. Sun, X. Xu, C. Zhang and X. He, Appl. Catal., B, 2019, 257, 117935.

21 J. Kosco, M. Bidwell, H. Cha, T. Martin, D. M. DeLongchamp, J. R. Durrant and I. McCulloch, Nat. Mater., 2020, 6597, 1-7.

22 C. M. Aitchison, R. S. Sprick and A. I. Cooper, J. Mater. Chem. A, 2019, 7, 2490-2496.

23 L. Wang, R. Fernandez-Teran, L. Tian, H. Chen and H. Tian, Angew. Chem., Int. Ed., 2016, 55, 12306-12310.

24 P. J. Tseng, C. L. Chang, C. H. Liao, M. L. Tsai and H. H. Chou, ACS Catal., 2018, 8, 7766-7772.

25 V. W. Lau, I. Moudrakovski, T. Botari, S. Weinberger, M. B. Mesch, V. Duppel, J. Senker, V. Blum and B. V. Lotsch, Nat. Commun., 2016, 7, 12165-12174.

26 Z. L. Enquan Jin, Q. Jiang, K. Geng, G. Li, X. Wang and D. Jiang, Chem, 2019, 5, 1632-1647.

27 T. Banerjee, F. Haase, G. Savasci, K. Gottschling, C. Ochsenfeld and B. V. Lotsch, J. Am. Chem. Soc., 2017, 139, 16228-16234.

28 B. P. Biswal, H. A. Vignolo-Gonzalez, J. Nuss, C. Ochsenfeld and B. V. Lotsch, J. Am. Chem. Soc., 2019, 141, 11082-11092.

29 W. Liu, W. Cheng, T. Yao and S. Wei, Angew. Chem., Int. Ed., 2017, 56, 9312-9317.

30 G. Zhao, Y. Sun, W. Zhou, X. Wang, K. Chang, G. Liu, H. Liu, T. Kako and J. Ye, Adv. Mater., 2017, 29, 1703258.

31 Y. Wan, L. Wang, H. Xu, X. Wu and J. Yang, J. Am. Chem. Soc., 2020, 142, 4508-4516.

32 Y. Zheng, Y. Jiao, Y. Zhu, L. H. Li, Y. Han, Y. Chen, A. Du, M. Jaroniec and S. Z. Qiao, Nat. Commun., 2014, 5, 37833791.

33 R. S. Sprick, L. Wilbraham, Y. Bai, P. Guiglion, A. Monti, R. Clowes, A. I. Cooper and M. A. Zwijnenburg, Chem. Mater., 2018, 30, 5733-5742.

34 P. Blonski, J. Tucek, Z. Sofer, M. Pumera, M. Otyepka and R. Zboril, J. Am. Chem. Soc., 2017, 139, 3171-3180.

35 I. Choudhuri, G. Bhattacharyya, S. Kumar and B. Pathak, J. Mater. Chem. C, 2016, 4, 11530-11539.

36 Y. Wu, X. Liu, X. Xiao and G. M. Wang, Nat. Commun., 2018, 9, 1-9.

37 C. Ling, L. Shi, Y. Ouyang, Q. Chen and J. Wang, Adv. Sci., 2016, 3, 1600180.

38 Y. Ouyang, Q. Li, L. Shi, C. Ling and J. Wang, J. Mater. Chem. A, 2018, 6, 2289-2294.
39 C. Ling, X. Niu, Q. Li, A. Du and J. Wang, J. Am. Chem. Soc., 2018, 140, 14161-14168.

40 W. Che, W. Cheng, T. Yao, F. Hu, Z. Pan, Z. Sun and S. Wei, J. Am. Chem. Soc., 2017, 139, 3021-3026.

41 C. F. Fu, X. Wu and J. Yang, Adv. Mater., 2018, 30, 1802106. 42 J. K. Norskov, T. Bligaard, J. Rossmeisl and C. H. Christensen, Nat. Chem., 2009, 1, 37-46.

43 J. Ran, G. Gao, F. T. Li, T. Y. Ma, A. Du and S. Z. Qiao, Nat. Commun., 2017, 8, 13907-13917.

44 P. Chen, T. Zhou, M. Zhang, Y. Tong, C. Zhong, N. Zhang, L. Zhang, C. Wu and Y. Xie, Adv. Mater., 2017, 29, 1701584.

45 Z. A. Lan, Y. Fang, Y. Zhang and X. Wang, Angew. Chem., Int. Ed., 2018, 57, 470-474.

46 K. Schwinghammer, M. B. Mesch, V. Duppel, C. Ziegler, J. Senker and B. V. Lotsch, J. Am. Chem. Soc., 2014, 136, 1730-1733.

47 L. Wang, X. Zheng, L. Chen, Y. Xiong and H. Xu, Angew. Chem., Int. Ed., 2018, 57, 3454-3458.

48 R. S. Sprick, C. M. Aitchison, M. A. Zwijnenburg and A. I. Cooper, J. Mater. Chem. A, 2018, 6, 11994-12003.

49 Y. Zhu, J. Chen, L. Shao, X. Xia, Y. Liu and L. Wang, Appl. Catal., B, 2020, 268, 118744.

50 B. Han, S. Liu, N. Zhang, Y. J. Xu and Z. R. Tang, Appl. Catal., $B, 2017,202,298-304$.

51 R. S. Sprick, J. X. Jiang, M. A. Zwijnenburg, D. J. Adams and A. I. Cooper, J. Am. Chem. Soc., 2015, 137, 3265-3270.

52 R. S. Sprick, B. Bonillo, M. A. Zwijnenburg, D. J. Adams and A. I. Cooper, Angew. Chem., Int. Ed., 2016, 55, 1824-1828.

53 D. J. Woods, R. S. Sprick, C. L. Smith, A. J. Cowan and A. I. Cooper, Adv. Energy Mater., 2017, 7, 1700479.

54 M. Sachs, R. S. Sprick, F. Blanc, M. A. Zwijnenburg, J. Nelson, J. R. Durrant and A. I. Cooper, Nat. Commun., 2018, 9, 49684979.

55 J. E. Nutting, M. Rafiee and S. S. Stahl, Chem. Rev., 2018, 118, 4834-4885.

56 C. Zhao, Z. Chen, J. Xu, Q. Liu, H. Xu, H. Tang, G. Li, Y. Jiang, F. Qu, Z. Lin and X. Yang, Appl. Catal., B, 2019, 256, 117867.

57 P. Kumar, E. Vahidzadeh, G. M. Bernard, V. K. Michaelis and K. Shankar, J. Am. Chem. Soc., 2019, 141, 5415-5436.

58 H. Huang, H. Yuan, K. P. F. Janssen, J. A. Steele and M. B. J. Roeffaers, ACS Energy Lett., 2018, 3, 755-759.

59 S. Bhattacharyya, F. Ehrat, P. Urban, A. S. Urban and J. K. Stolarczyk, Nat. Commun., 2017, 8, 1401-1410.

60 S. Wei, F. Zhang, W. Zhang, P. Qiang, K. Yu, X. Fu, D. Wu, S. Bi and F. Zhang, J. Am. Chem. Soc., 2019, 141, 1427214279.

61 X. Gao, C. Shu, C. Zhang, W. Ma, S.-B. Ren, F. Wang, Y. Chen, J. H. Zeng and J. X. Jiang, J. Mater. Chem. A, 2020, 8, 24042411.

62 Z. Zhou, P. Peng and Z. Xiang, Sci. Bull., 2018, 63, 369-375.

63 J. Cheng, G. Liao, L. Chen and S. Y. Liu, J. Mater. Chem. A, 2020, 8, 5890-5899.

64 Q. Ruan, J. Xie, Y. Wang, C. J. Carmalt and J. Tang, Angew. Chem., Int. Ed., 2017, 56, 8221-8225.

65 M. Shi, J. Li, X. Jin, E. A. Pidko, R. Li and C. Li, Angew. Chem., Int. Ed., 2019, 59, 6590-6595. 